\title{
THEMIS observations of the magnetopause electron diffusion region: Large amplitude waves and heated electrons
}

\author{
Xiangwei Tang, ${ }^{1}$ Cynthia Cattell, ${ }^{1}$ John Dombeck, ${ }^{1}$ Lei Dai, ${ }^{1}$ Lynn B. Wilson III, ${ }^{2}$ \\ Aaron Breneman, ${ }^{1}$ and Adam Hupach ${ }^{1}$ \\ Received 14 April 2013; revised 9 May 2013; accepted 14 May 2013; published 18 June 2013.
}

[1] We present the first observations of large amplitude waves in a well-defined electron diffusion region based on the criteria described by Scudder et al. [2012] at the subsolar magnetopause using data from one Time History of Events and Macroscale Interactions during Substorms (THEMIS) satellite. These waves identified as whistler mode waves, electrostatic solitary waves, lower hybrid waves, and electrostatic electron cyclotron waves, are observed in the same $12 \mathrm{~s}$ waveform capture and in association with signatures of active magnetic reconnection. The large amplitude waves in the electron diffusion region are coincident with abrupt increases in electron parallel temperature suggesting strong wave heating. The whistler mode waves, which are at the electron scale and which enable us to probe electron dynamics in the diffusion region were analyzed in detail. The energetic electrons $(\sim 30 \mathrm{keV})$ within the electron diffusion region have anisotropic distributions with $\mathrm{T}_{e \perp} / \mathrm{T}_{e \|}>1$ that may provide the free energy for the whistler mode waves. The energetic anisotropic electrons may be produced during the reconnection process. The whistler mode waves propagate away from the center of the "X-line" along magnetic field lines, suggesting that the electron diffusion region is a possible source region of the whistler mode waves. Citation: Tang, X., C. Cattell, J. Dombeck, L. Dai, L. B. Wilson III, A. Breneman, and A. Hupach (2013), THEMIS observations of the magnetopause electron diffusion region: Large amplitude waves and heated electrons, Geophys. Res. Lett., 40, 2884-2890, doi:10.1002/ grl.50565.

\section{Introduction}

[2] Magnetic reconnection is considered to be an important energy conversion process that occurs in a variety of plasma environments. At the Earth's magnetopause, it facilitates the entry of solar wind plasma and electromagnetic energy into the magnetosphere. Strong wave turbulence covering a broad range of frequencies has been frequently observed near the reconnection site [Retinò et al., 2006; Khotyaintsev et al., 2006; Cattell et al., 2005]. More specifically, these wave modes include the whistler mode (WH) waves [Deng and Matsumoto, 2001; Petkaki et al., 2006], electrostatic solitary waves (ESWs) [Farrell et al., 2002; Matsumoto et al., 2003],

\footnotetext{
${ }^{1}$ School of Physics and Astronomy, University of Minnesota, Minneapolis, Minnesota, USA.

${ }^{2}$ Heliospheric Physics Laboratory, NASA Goddard Space Flight Center, Greenbelt, Maryland, USA.

Corresponding author: X. Tang, School of Physics and Astronomy, University of Minnesota, 116 Church St. S.E., Minneapolis, MN 55455, USA. (xtangphysics@gmail.com)

(C2013. American Geophysical Union. All Rights Reserved. 0094-8276/13/10.1002/grl.50565
}

lower hybrid (LH) waves [Cattell and Mozer, 1986; Bale et al., 2002], kinetic Alfvén waves [Chaston et al., 2005], and Langmuir/upper hybrid waves [Farrell et al., 2002]. The effect of different wave modes on the reconnection process has been a problem of long-standing interest-for their role in anomalous resistivity, particle acceleration, energy transport, and formation of reconnection sites [Huba et al., 1977; Labelle and Treumann, 1988; Treumann et al., 1991; Drake et al., 2003; Roytershteyn et al., 2012].

[3] The physics of reconnection occurs on the larger spatial scale (ion diffusion region) and on the smaller scale (electron diffusion region) that are associated with the ion and electron dynamics, respectively. Properties of collisionless asymmetric reconnection have been compared to those of symmetric reconnection on ion-scale physics [Pritchett, 2008] and electron-scale physics [Mozer and Pritchett, 2011, and references therein]. Simulations have been performed to investigate the effects of diamagnetic drift [Swisdak et al., 2003], guide magnetic field [Pritchett and Mozer, 2009], and dissipation mechanism [Aunai et al., 2013] on collisionless asymmetric reconnection. The efficiency of collisionless asymmetric reconnection is controlled by the physics in the electron diffusion region (EDR). Observations of EDRs have been made by Polar at the subsolar magnetopause [Mozer et al., 2003], by Wind in the magnetotail [Øieroset et al., 2001], and by Cluster in the magnetosheath [Phan et al., 2007]. Recent simulations and observations of EDRs during collisionless antiparallel reconnection in Earth's magnetotail [ $\mathrm{Ng}$ et al., 2011] report that the diffusion region is characterized by a narrow extended layer containing electron jets. It is shown that the jets in the layer are driven by electron pressure anisotropy $\mathrm{P}_{e \|} \gg \mathrm{P}_{e \perp}$, and the anisotropy is responsible for the structure of the EDR [ $\mathrm{Ng}$ et al., 2011]. Mozer [2005] has identified EDRs on the basis of the nonzero parallel electric field, a large perpendicular electric field compared to the reconnection electric field, a large electromagnetic energy conversion rate and accelerated electrons, and a topological boundary that separates regions having different $\mathbf{E} \times \mathbf{B} / B^{2}$ flows with thickness of the order of the electron skin depth. In this paper, we concentrate on a specific way to identify the EDR described by Scudder et al. [2012] who report spatially resolved diagnostic signatures of a demagnetized EDR observed by Polar at the Earth's magnetopause. The criteria for being in an EDR should be electron specific since electrons are locally disrupted in the EDR. The four dimensionless scalar diagnostics that were used to find the EDR are peak electron thermal Mach numbers $\mathrm{M}_{e \perp} \equiv\left|\mathbf{U}_{e}\right| /\left\langle w_{e \perp}\right\rangle$ $>1.5$ (where $\mathbf{U}_{e}$ represents electron bulk velocity and $\left\langle w_{e} \perp\right\rangle$ is the electron thermal speed derived from the average 
perpendicular temperatures), electron temperature anisotropy $\mathrm{An}_{e} \equiv \mathrm{T}_{e \|} /\left\langle T_{e \perp}\right\rangle>7$, calibrated agyrotropy of electron pressure tensor $A \varnothing_{\mathrm{e}}=2|1-\alpha| /(1+\alpha)>1\left(\right.$ where $\left.\alpha \equiv \mathrm{P}_{e \perp, 1} / \mathrm{P}_{e \perp, 2}\right)$, and strong ( $\gtrsim 150 \mathrm{eV}$ ) increases in electron temperature [Scudder et al., 2012]. The electron pressure agyrotropy is a measure of the departure of the pressure tensor from cylindrical symmetry about the local magnetic field and it reflects the demagnetization of the thermal electrons, which enables collisionless reconnection.

[4] This paper focuses on the WH waves, which are an important candidate for the anomalous resistivity, particle acceleration, and heating. WH waves may be driven unstable by superthermal electrons with temperature anisotropies of $\mathrm{T}_{e \perp} / \mathrm{T}_{e \|}>1$ in the magnetosphere [Kennel and Petschek, 1966] and current-driven plasma instabilities [Gurnett et al., 1976] or energetic electron beams [Zhang et al., 1999] in the magnetotail. WH waves are one of the most ubiquitous wave modes observed in space plasmas. Observations of WH waves at the Earth's magnetopause have been made by Deng and Matsumoto [2001]. Electron anisotropy, due to compression of the magnetopause or LH drift waves, may be the generation mechanism of $\mathrm{WH}$ waves in the magnetopause current sheet [Karimabadi et al., 2004]. WH waves in the EDR may play a significant role in the microphysics of reconnection as they are excited on electron scales. It is believed that WH waves in the magnetopause current sheet may affect the instability of the current sheet to reconnection via tearing. The generation of the outof-plane component of the magnetic field is suggested to be a signature of whistler-mediated reconnection [Mandt et al., 1994]. It has also been suggested that the strongest whistler emissions are observed on the most recently opened magnetospheric flux tubes due to magnetic reconnection [Vaivads et al., 2007]. One recent simulation study concludes that WH waves do not control the dissipation processes of reconnection but are generated as a result of the reconnection processes [Fujimoto and Sydora, 2008]. In this paper, we present an example of a reconnection event at the subsolar magnetopause observed by Time History of Events and Macroscale Interactions during Substorms (THEMIS). In section 2, we describe the data sets and analysis techniques. In section 3, we show the observations. Finally, we discuss the conclusions of our study in section 4.

\section{Data Sets and Analysis}

[5] The THEMIS mission consists of five identically instrumented spacecraft. The Electric Field Instrument (EFI) measures three components of the electric field [Bonnell et al., 2008]. The instrument provides continuous coverage at 128 samples/s in survey mode and waveform captures at 8192 samples/s in wave burst mode. The Magnetic Fields Experiment (MFE) includes a fluxgate magnetometer (FGM), which measures DC magnetic field with a sampling rate of $128 \mathrm{samples} / \mathrm{s}$ in the high rate mode or $4 \mathrm{samples} / \mathrm{s}$ in the low rate mode, and a search coil magnetometer, (SCM) which measures magnetic fluctuations sampled at 8192 samples/s in the burst mode [Roux et al., 2008]. Particle data are measured by the Electrostatic Analyzer (ESA) [McFadden et al., 2008] and by the Solid State Telescope (SST) [Angelopoulos, 2008]. The ESA measures plasma over the energy range of a few $\mathrm{eV}$ up to $30 \mathrm{keV}$ for electrons and $25 \mathrm{keV}$ for ions. The
SST measures the distribution functions of superthermal particles within the energy range from $25 \mathrm{keV}$ to $6 \mathrm{MeV}$ for electrons and $900 \mathrm{keV}$ for ions. Because the measured quasistatic electric field component along the spin axis has large uncertainty due to the short boom along the spin axis, we use $\mathbf{E} \cdot \mathbf{B}=0$ to determine the electric field used to calculate the $\mathbf{E} \times \mathbf{B} / B^{2}$ velocity. The coordinate systems used in this paper include geocentric solar magnetospheric (GSM) coordinates and field-aligned coordinates (FAC). The FAC is defined in the following way: The positive $Z$ axis points in the direction of the magnetic field at the spacecraft's location. The positive $X$ axis lies in the plane of the magnetic field line passing through the spacecraft's location, perpendicular to the $Z$ axis, and points inward (toward the inside of the field line). The positive $Y$ axis completes the orthogonal right-handed system. Waveforms are analyzed dynamically in time and frequency using Morlet wavelet transform. The wave vector is determined using Minimum Variance Analysis (MVA) on band pass-filtered three magnetic field components.

\section{Observations}

[6] Figure 1 shows a $5.5 \mathrm{~min}$ interval of the field and plasma observations made by probe $\mathrm{E}$ of the THEMIS mission on 27 August 2009. The boundary normal direction (determined from MVA on the ambient magnetic field) was $[0.99,0.015,-0.12]$ in GSM coordinates and almost identical to the GSM-X direction, consistent with the spacecraft being near the subsolar point (indicated by the position parameters at the bottom of Figure 1). The spacecraft travels from the outer magnetosphere (SP) through the magnetopause (MP, indicated by two light green shaded bands) into the magnetosheath ( $\mathrm{SH})$. The purple shaded band shows a $\sim 12 \mathrm{~s}$ interval of magnetic burst data capture.

[7] The magnetopause crossing can be seen in the change in $\mathrm{Bz}$ from positive to negative (Figure 1a). The differential energy flux of electrons in Figure $1 \mathrm{j}$ shows that in the magnetosphere, where $\mathrm{Bz}$ was positive, higher-energy electrons were encountered; while in the magnetosheath, where $\mathrm{Bz}$ was negative, lower-energy electrons were measured. The spacecraft passed from the lower plasma density magnetosphere to the high-density (factor of 100) magnetosheath via a region of mixed magnetosheath/magnetospheric plasmas comprising the low-latitude boundary layer as shown in Figure 1g. This observation is evidence for the transport of solar wind plasma across the magnetopause. The presence of accelerated plasma flow is seen through the magnetopause current sheet as shown in Figures 1d and 1e. The spacecraft crossed the magnetopause south of the separator, as suggested by the negative GSM-Z component of ion flow velocity and the result of Walén test [Sonnerup et al., 1981]. Based on the prediction of the magnetopause reconnection model being a rotational discontinuity [Paschmann et al., 1979], the Walén test states that the observed flow velocity change between a point in the magnetopause and a reference point in the adjacent magnetosheath equals the predicted modified Alfvén velocity change. The ratios of magnitudes between the observed flow velocity change and the theoretically predicted modified Alfvén velocity change range from 0.30 to 0.66 , which is reasonable within the range of uncertainty. The angle deviations 
TANG ET AL.: OBSERVATIONS OF WAVES

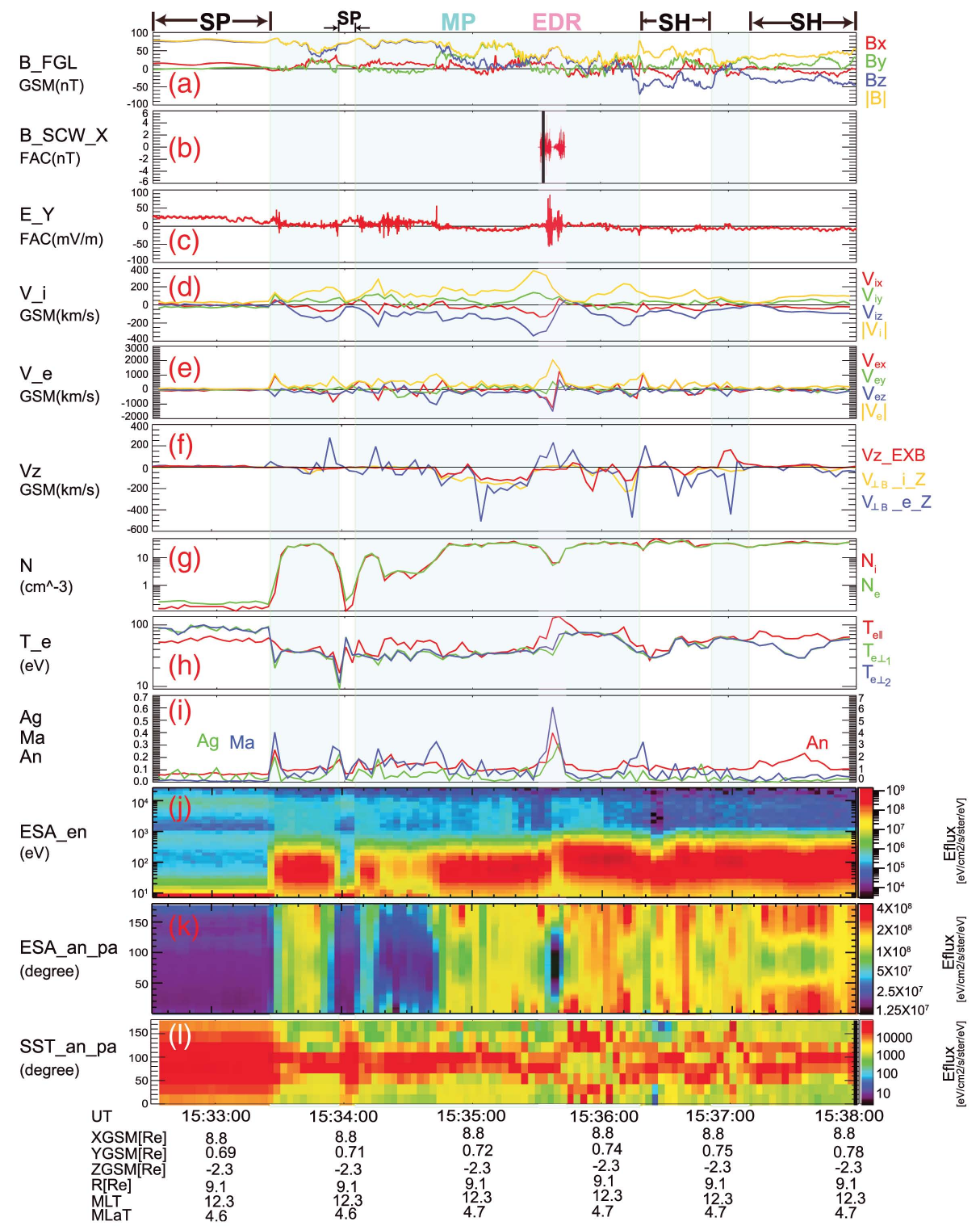

Figure 1. A reconnection event at the subsolar magnetopause observed by THEMIS-E on 27 August 2009. (a) 4 samples/s magnetic field data in GSM. (b) Perpendicular $X$ component of the burst magnetic field at $8192 \mathrm{samples} / \mathrm{s}$ in FAC. (c) Perpendicular $Y$ component of the electric field at 128 samples/s in FAC. (d and e) Ion and electron bulk flow velocity in GSM, respectively. (f) Comparisons of GSM-Z component of the $\mathbf{E} \times \mathbf{B} / B^{2}$ velocity with the GSM-Z component of ion and electron perpendicular flow velocity with respect to the ambient magnetic field, respectively. (g) Ion and electron densities. (h) Electron temperatures. (i) Electron agyrotropy and Mach number (scale to the left) and temperature anisotropy (scale to the right). (j) Differential energy flux for electrons measured by ESA. ( $k$ and l) Electron pitch angle spectra for lower-energy $(7 \mathrm{eV}-26 \mathrm{keV})$ electrons measured by ESA and higher-energy $(31-719 \mathrm{keV})$ electrons measured by SST, respectively.

between the observed and the predicted velocity changes are almost $180^{\circ}$ for this event. This antiparallel relation indicates that the normal magnetic field component $\mathrm{Bn}$ should be positive and thus the spacecraft crossed south of the separator [Sonnerup et al., 1981]. This result is consistent with the positive polarity of the GSM-X component of the magnetic field, which is an approximation for Bn shown in Figure 1a (around 15:35:30 UT). The encounter with the magnetopause current sheet is associated with fast ion jetting consistent with the Walén relation and fast electron flows, indicating that reconnection is occurring. Magnetic reconnection is generally considered to be the primary mechanism through which transport of plasma and energy across the magnetopause occurs.

\subsection{Identification of Electron Diffusion Region}

[8] Enhanced wave activity can be seen associated with the magnetopause crossing from Figures 1a (ambient magnetic field), $1 \mathrm{~b}$ (burst magnetic field), and 1c (electric fluctuations). We note that the electric fluctuations maximize during the magnetic burst interval. During this interval, electron flow speed (Figure 1e), anisotropy, agyrotropy, and Mach number (Figure 1i) also maximize. These enhanced amplitudes are coincident with abrupt increases in electron parallel temperature $\mathrm{T}_{e \|}$ shown in Figure $1 \mathrm{~h}$ suggesting strong wave heating. As will be discussed in more detail in section 3.3, the observed intense waves may provide the observed electron heating. All these features, along with the fact that the electron perpendicular flow 


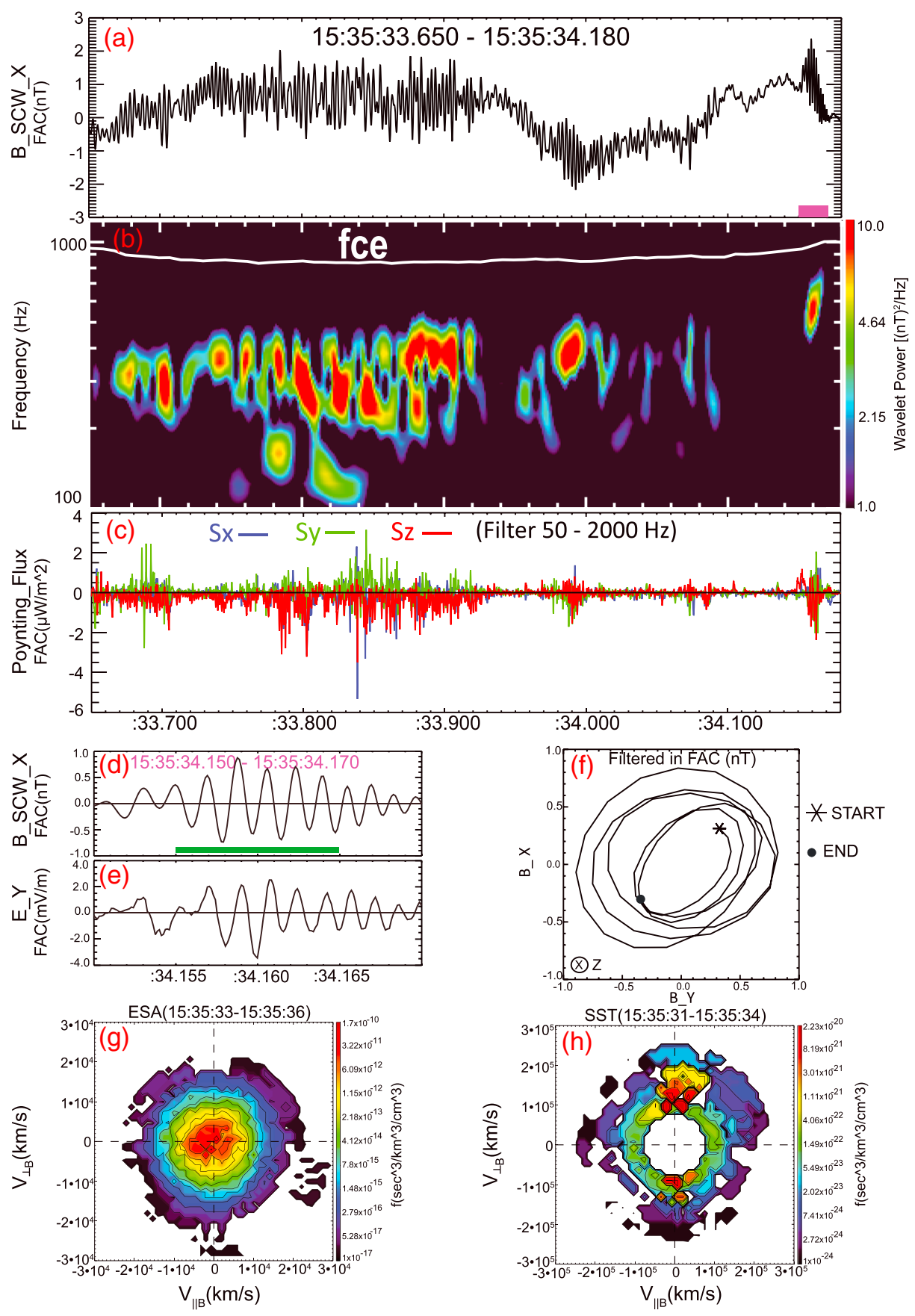

Figure 2. Example of WH waves within the EDR. (a, b, and c) A $0.53 \mathrm{~s}$ interval in FAC of perpendicular $X$ component of the burst magnetic field, associated wavelet power spectrum, and whistler Poynting flux, respectively. ( $d$ and e) Expanded views of the filtered whistler waveforms of the perpendicular $X$ component of the burst magnetic field and the perpendicular $Y$ component of the electric field over the time interval indicated by the purple bar in Figure 2a. (f) Hodogram of the filtered burst magnetic field waveforms in FAC for the interval indicated by the green bar in Figure 2d. The black star and dot mark the beginning and ending of the wave field, respectively. ( $g$ and $h$ ) Distribution functions of lower-energy electrons (up to $20 \mathrm{keV})$ measured by ESA and higher-energy electrons $(20-700 \mathrm{keV})$ measured by SST observed at times close to the WH waves, respectively. The horizontal axis is parallel to the ambient magnetic field and the bulk velocity defines the plane.

velocity is not consistent with the $\mathbf{E} \times \mathbf{B} / B^{2}$ velocity during the magnetic burst interval (Figure 1f), provide evidence for the detection of an EDR. These signatures are consistent with the simulation and observations of Scudder et al. [2012]. It should be noted that the small peaks in the electron thermal parameters correspond to entries of topological boundaries of the magnetic field. For example, the peaks around 15:33:30
UT are due to the entry of magnetopause boundary layer. A density cavity can be seen in Figure $1 \mathrm{~g}$ (purple shaded band). The density inside the density cavity decreases to $16 \%$ of the magnetopause boundary level $\left(30 \mathrm{~cm}^{-3}\right)$, which might indicate the center of the EDR. This density cavity is not a signature of magnetospheric separatrix as the density inside the density cavity does not drop with respect to its magnetospheric level. 


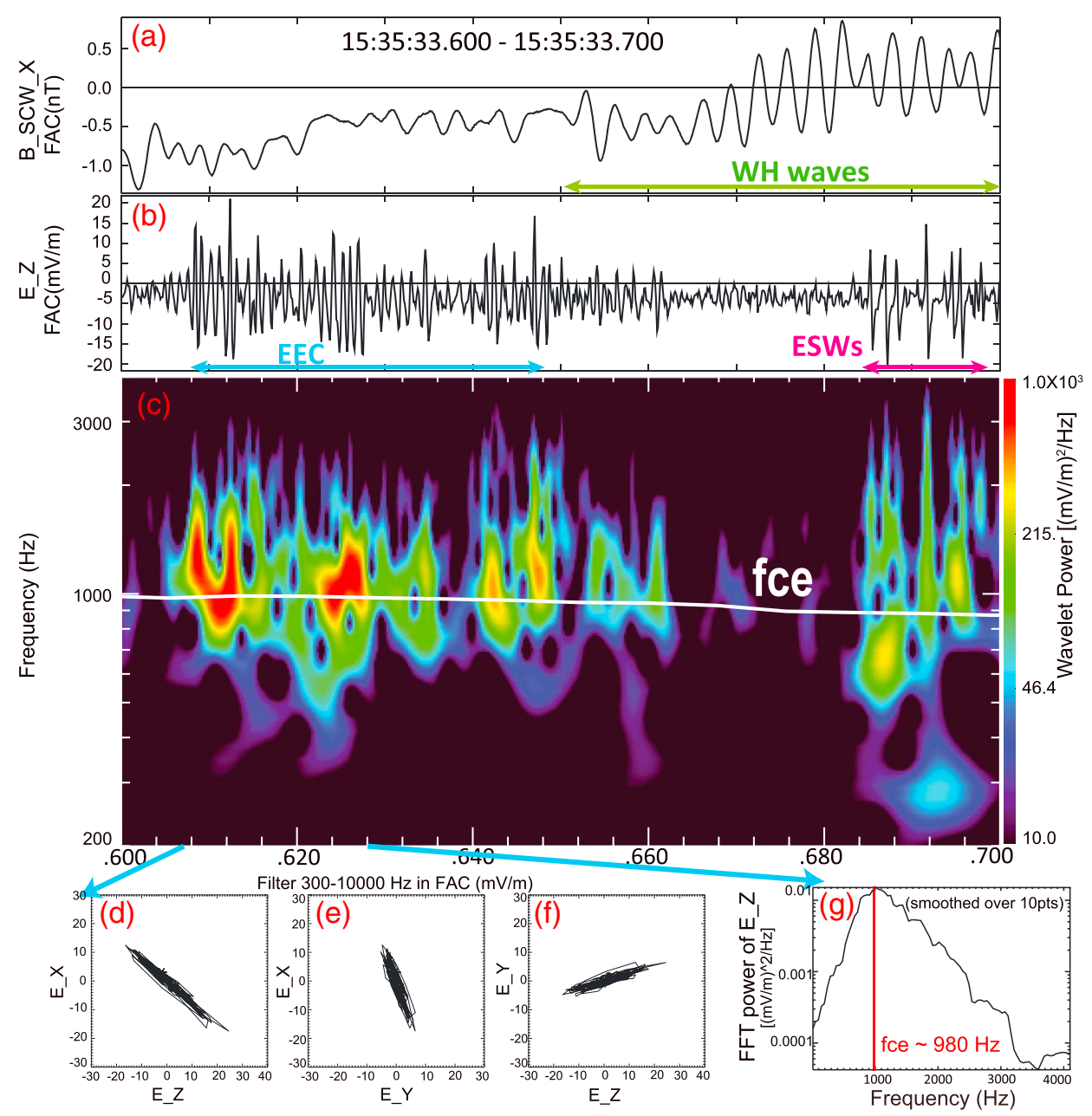

Figure 3. Example of electrostatic waves within the EDR. (a, b, and c) A $0.1 \mathrm{~s}$ interval of the perpendicular $X$ component of the burst magnetic field, the parallel $Z$ component of the electric field waveform capture and the associated wavelet power spectrum of the parallel electric field in FAC, respectively. (d, e, and f) Hodograms of the electric field waveforms with an interval indicated by the light blue arrows below Figure 3c. (g) Fourier wave power versus frequency with the same time interval as the hodograms.

From the deviations of ion perpendicular flow velocity from the $\mathbf{E} \times \mathbf{B} / \mathrm{B}^{2}$ velocity (Figure $1 \mathrm{f}$ ), it can be seen that the ion diffusion region was encountered probably during the following two time intervals: 15:34:40-15:36:20 UT and 15:36:50-15:37:10 UT.

\subsection{Distributions of Electrons}

[9] Figures $1 \mathrm{k}$ and 11 show the electron pitch angle spectra for lower-energy electrons measured by ESA and higherenergy electrons measured by SST, respectively. The magnetic field (Figure 1a) and electric field (Figure 1c) fluctuations enhance in the magnetopause boundary layer and in the magnetosheath with field-aligned and counter-streaming lower-energy electrons as shown in Figure 1k. However, the higher-energy electron pitch angle (Figure 11) enhances around $90^{\circ}$ in the boundary layer and in the magnetosheath, especially during the purple shaded magnetic burst interval. Distinct from the electron distributions in the boundary layer and in the magnetosheath, the higher-energy electron pitch angle enhances at $0^{\circ}$ and $180^{\circ}$, and the lower-energy electrons are more isotropic near the current sheet center around 15:36:00 UT and 15:37:00 UT. The lower-energy electrons in the identified EDR in Figure $1 \mathrm{k}$ can be seen streaming away from the "X-line" (antiparallel to the local magnetic field). These electrons are associated with Hall current parallel to the magnetic field. This indicates that the identified EDR is close to the "X-line."

\subsection{Observations of Waves}

[10] Figure 2 shows an example of the identified WH waves at the time indicated by a black vertical line in Figure 1b. It can be seen from Figures $2 a$ and $2 b$ that the waves have frequencies from 0.1 to $0.6 \mathrm{f}_{\mathrm{ce}}$ (electron cyclotron frequency) with amplitudes up to $3 \mathrm{nT}$ (peak-peak). As can be seen from Figure 2c, the wave Poynting flux is mostly antiparallel to the ambient magnetic field. The wave vector $(k=[-0.23$, $-0.09,0.97]$ in FAC), determined from MVA on band passfiltered wave magnetic field, is nearly along the background magnetic field. The wave propagation angle with respect to the ambient magnetic field $\theta_{\mathrm{kB}}$ is determined as $\sim 166^{\circ}$ since the Poynting flux is mostly antiparallel to the magnetic field. An expanded view of the WH waves can be seen in Figures $2 \mathrm{~d}$ and $2 \mathrm{e}$, which respectively show the filtered $(200-2000 \mathrm{~Hz})$ waveforms of the burst magnetic and electric field data over the time interval indicated by the purple bar 
in Figure 2a. Figure $2 \mathrm{f}$ shows that the WH waves are circularly right-handed polarized with respect to the ambient magnetic field as expected. The electron distribution functions observed at times close to and/or concurrently with the WH waves are shown in Figures $2 \mathrm{~g}$ and $2 \mathrm{~h}$. The lower-energy electrons $(\sim 100 \mathrm{eV})$ shown in Figure $2 \mathrm{~g}$ have anisotropic distributions with $\mathrm{T}_{e \perp} / \mathrm{T}_{e \|}<1$. However, the energetic electrons $(\sim 30 \mathrm{keV})$ shown in Figure $2 \mathrm{~h}$ have anisotropic distributions with a larger population moving in perpendicular direction. Broad-banded emissions with strong electric $(\sim 10 \mathrm{mV} / \mathrm{m})$ and magnetic $(\sim 40 \mathrm{nT})$ field fluctuations below ion cyclotron frequency $(\sim 1 \mathrm{~Hz})$, and electric $(\sim 30 \mathrm{mV} / \mathrm{m})$ and magnetic $(\sim 20 \mathrm{nT})$ field fluctuations below LH frequency $(\sim 30 \mathrm{~Hz})$ are also detected during this magnetopause crossing (not shown). These intense wave emissions may provide the observed electron heating associated with the magnetopause crossing.

[11] Figure 3 shows examples of electrostatic waves at a time preceding the waves in Figure 2 by $0.05 \mathrm{~s}$. WH waves at a frequency of $\sim 0.3 \mathrm{f}_{\text {ce }}$ are observed in the magnetic fluctuations in Figure $3 \mathrm{a}$ from 15:35:33.650 UT to 15:35:33.700 UT. This time interval overlaps with that of the ESWs, suggesting a possible coupling of WH waves and ESWs. ESWs (up to $30 \mathrm{mV} / \mathrm{m}$ ) indicated by the magenta arrows in Figure $3 \mathrm{~b}$ have a broad spectrum, which extends from 200 to $3000 \mathrm{~Hz}$ shown in Figure 3c. The high-frequency electrostatic waves (up to $35 \mathrm{mV} / \mathrm{m}$ ) labeled by light blue arrows in Figure $3 \mathrm{~b}$ have power that peaks at $\mathrm{f}_{\text {ce }}$, which can be seen from both the wavelet power spectrum in Figure $3 \mathrm{c}$ and the Fourier power in Figure $3 \mathrm{~g}$. During this time interval, there is no power in the wave magnetic field at $f_{c e}$ (not shown). This wave mode is linearly polarized, as shown in the hodograms in Figures 3d, $3 \mathrm{e}$, and $3 \mathrm{f}$ in FAC with an interval indicated by the light blue arrows below Figure 3c. Occasionally, these high-frequency emissions are seen associated with clear harmonics, possibly suggesting electrostatic electron cyclotron waves.

\section{Discussion and Conclusions}

[12] We have presented the first observations of intense waves in the EDR in a subsolar magnetopause reconnection region. The identification of the EDR in this event is based on the occurrence of signatures of strong electron heating, large electron thermal anisotropy, agyrotropy, and Mach number and electron velocity not consistent with the $\mathbf{E} \times \mathbf{B}$ velocity, consistent with the simulation and observations of Scudder et al. [2012]. The lower-energy electrons $(\sim 100 \mathrm{eV})$ with anisotropic distributions of $\mathrm{T}_{e \perp} / \mathrm{T}_{e} \| 1$ within the EDR may have been heated by the observed waves with frequency below the LH frequency, consistent with the suggestion that LH waves lead to electron heating in the parallel direction [Cairns and McMillan, 2005].

[13] We identified intense WH waves inside the EDR. This is inconsistent with reported simulation results that indicated WH waves are only driven downstream of an EDR [Fujimoto and Sydora, 2008]. The WH waves seen by THEMIS in the EDR propagate almost antiparallel to the ambient magnetic field, and the Poynting flux indicates that the WH waves propagate away from the center of the "X-line" along magnetic field lines. The observed electron temperature anisotropy of $\mathrm{T}_{e \perp} / \mathrm{T}_{e} \| 1$ for energies above $20 \mathrm{keV}$ may be the source of free energy for the generation of the WH waves. The energetic electron anisotropy may be produced by adiabatic heating in the perpendicular direction as the locally intensified magnetic field can accelerate electrons in the perpendicular direction [Fujimoto and Sydora, 2008]. On the field lines directly connected to the EDR, the energetic electron anisotropy may also be due to higher-energy fieldaligned electrons (accelerated by the reconnection process) being lost to the magnetosheath [Stenberg et al., 2005]. WH waves can scatter the electrons in pitch-angle distribution and relax the temperature anisotropy. Studies of large amplitude whistlers in the inner magnetosphere have provided evidence for rapid scattering and/or energization [Cattell et al., 2008]. WH waves may play a significant role in the microphysics of reconnection through the enabling of a current sheet instability, the decoupling of electrons, the acceleration and heating of particles, and the transport of energy away from the reconnection region.

[14] A possible coupling of electrostatic electron cyclotron waves and ESWs with WH waves is often seen during magnetopause reconnection. The growth of the electrostatic waves may reduce the electron temperature anisotropy and reduce the growth rate of $\mathrm{WH}$ waves. The physics of wave coupling process is important to understand the effect of wave-wave interactions on the reconnection process and will be investigated in a future study. This study provides further evidence that the plasma waves can play a significant role in the microphysics of magnetic reconnection at the Earth's magnetopause.

[15] Acknowledgments. At the University of Minnesota, this work was supported by NNX08AF28, NNX13AE16G, and a contract from APL for the development of RBSP/EFW. The authors acknowledge NASA contract NAS5-02099 and V. Angelopoulos for use of data from the THEMIS Mission, specifically: J. W. Bonnell and F. S. Mozer for use of EFI data; D. Larson and R. P. Lin for use of SST data; C. W. Carlson and J. P. McFadden for use of ESA data; A. Roux and O. LeContel for use of SCM data; and K. H. Glassmeier, U. Auster, and W. Baumjohann for the use of FGM data provided under the lead of the Technical University of Braunschweig and with financial support through the German Ministry for Economy and Technology and the German Center for Aviation and Space (DLR) under contract 50 OC 0302 . The authors are grateful for discussion and comments from S. Thaller, K. Kersten, and C. Colpitts.

[16] The Editor thanks Ruben Juanes and an anonymous reviewer for their assistance in evaluating this paper.

\section{References}

Angelopoulos, V. (2008), The THEMIS mission, Space Sci. Rev., 141, 5-34, doi:10.1007/s11214-008-9336-1.

Aunai, N., M. Hesse, C. Black, R. Evans, and M. Kuznetsova (2013), Influence of the dissipation mechanism on collisionless magnetic reconnection in symmetric and asymmetric current layers, Phys. Plasmas, 20(4), 042901, doi:10.1063/1.4795727.

Bale, S. D., F. S. Mozer, and T. Phan (2002), Observation of lower hybrid drift instability in the diffusion region at a reconnecting magnetopause, Geophys. Res. Lett., 29(24), 2180, doi:10.1029/2002GL016113.

Bonnell, J. W., F. S. Mozer, G. T. Delory, A. J. Hull, R. E. Ergun, C. M. Cully, V. Angelopoulos, and P. R. Harvey (2008), The Electric Field Instrument (EFI) for THEMIS, Space Sci. Rev., 141, 303-341, doi:10.1007/s11214-008-9469-2.

Cairns, I. H., and B. F. McMillan (2005), Electron acceleration by lower hybrid waves in magnetic reconnection regions, Phys. Plasmas, 12(10), 102110, doi:10.1063/1.2080567.

Cattell, C. A., and F. S. Mozer (1986), Experimental determination of the dominant wave mode in the active near-Earth magnetotail, Geophys. Res. Lett., 13, 221-224, doi:10.1029/GL013i003p00221.

Cattell, C., et al. (2005), Cluster observations of electron holes in association with magnetotail reconnection and comparison to simulations, J. Geophys. Res., 110, A01211, doi:10.1029/2004JA010519.

Cattell, C., et al. (2008), Discovery of very large amplitude whistler-mode waves in Earth's radiation belts, Geophys. Res. Lett., 35, L01105, doi:10.1029/2007GL032009.

Chaston, C. C., et al. (2005), Drift-kinetic Alfvén waves observed near a reconnection X line in the Earth's magnetopause, Phys. Rev. Lett., 95, 065002, doi:10.1103/PhysRevLett.95.065002. 
Deng, X. H., and H. Matsumoto (2001), Rapid magnetic reconnection in the Earth's magnetosphere mediated by whistler waves, Nature, 410, 557-560, doi:10.1038/35069018.

Drake, J. F., M. Swisdak, C. Cattell, M. A. Shay, B. N. Rogers, and A. Zeiler (2003), Formation of electron holes and particle energization during magnetic reconnection, Science, 299, 873-877, doi:10.1126/science.1080333.

Farrell, W. M., M. D. Desch, M. L. Kaiser, and K. Goetz (2002), The dominance of electron plasma waves near a reconnection X-line region, Geophys. Res. Lett., 29(19), 1902, doi:10.1029/2002GL014662.

Fujimoto, K., and R. D. Sydora (2008), Whistler waves associated with magnetic reconnection, Geophys. Res. Lett., 35, L19112, doi:10.1029/ 2008GL035201.

Gurnett, D. A., L. A. Frank, and R. P. Lepping (1976), Plasma waves in the distant magnetotail, J. Geophys. Res., 81, 6059-6071, doi:10.1029/ JA081i034p06059.

Huba, J. D., N. T. Gladd, and K. Papadopoulos (1977), The lower-hybriddrift instability as a source of anomalous resistivity for magnetic field line reconnection, Geophys. Res. Lett., 4, 125-126, doi:10.1029/ GL004i003p00125.

Karimabadi, H., W. Daughton, and K. B. Quest (2004), Role of electron temperature anisotropy in the onset of magnetic reconnection, Geophys. Res. Lett., 31, L18801, doi:10.1029/2004GL020791.

Kennel, C. F., and H. E. Petschek (1966), Limit on stably trapped particle fluxes, J. Geophys. Res., 71, 1-28, doi:10.1029/JZ071i001p00001.

Khotyaintsev, Y. V., A. Vaivads, A. Retinò, M. André, C. J. Owen, and H. Nilsson (2006), Formation of inner structure of a reconnection separatrix region, Phys. Rev. Lett., 97(20), 205003, doi:10.1103/ PhysRevLett.97.205003.

Labelle, J., and R. A. Treumann (1988), Plasma waves at the dayside magnetopause, Space Sci. Rev., 47, 175-202, doi:10.1007/BF00223240.

Mandt, M. E., R. E. Denton, and J. F. Drake (1994), Transition to whistler mediated magnetic reconnection, Geophys. Res. Lett., 21, 73-76, doi:10.1029/93GL03382.

Matsumoto, H., X. H. Deng, H. Kojima, and R. R. Anderson (2003), Observation of electrostatic solitary waves associated with reconnection on the dayside magnetopause boundary, Geophys. Res. Lett., 30(6), 1326, doi:10.1029/2002GL016319.

McFadden, J. P., C. W. Carlson, D. Larson, M. Ludlam, R. Abiad, B. Elliott, P. Turin, M. Marckwordt, and V. Angelopoulos (2008), The THEMIS ESA plasma instrument and in-flight calibration, Space Sci. Rev., 141, 277-302, doi:10.1007/s11214-008-9440-2.

Mozer, F. S. (2005), Criteria for and statistics of electron diffusion regions associated with subsolar magnetic field reconnection, J. Geophys. Res. 110, A12222, doi:10.1029/2005JA011258.

Mozer, F. S., and P. L. Pritchett (2011), Electron physics of asymmetric magnetic field reconnection, Space Sci. Rev., 158, 119-143, doi:10.1007/ s11214-010-9681-8.

Mozer, F. S., S. D. Bale, T. D. Phan, and J. A. Osborne (2003), Observations of electron diffusion regions at the subsolar magnetopause, Phys. Rev. Lett., 91, 245002, doi:10.1103/PhysRevLett.91.245002.

Ng, J., J. Egedal, A. Le, W. Daughton, and L.-J. Chen (2011), Kinetic structure of the electron diffusion region in antiparallel magnetic reconnection, Phys. Rev. Lett., 106(6), 065002, doi:10.1103/PhysRevLett.106.065002.

Øieroset, M., T. D. Phan, M. Fujimoto, R. P. Lin, and R. P. Lepping (2001), In situ detection of collisionless reconnection in the Earth's magnetotail, Nature, 412, 414-417, doi:10.1038/35086520.
Paschmann, G., et al. (1979), Plasma acceleration at the earth's magnetopause-Evidence for reconnection, Nature, 282, 243-246, doi:10.1038/ $282243 \mathrm{a} 0$.

Petkaki, P., M. P. Freeman, and A. P. Walsh (2006), Cluster observations of broadband electromagnetic waves in and around a reconnection region in the Earth's magnetotail current sheet, Geophys. Res. Lett., 33, L16105, doi:10.1029/2006GL027066.

Phan, T. D., J. F. Drake, M. A. Shay, F. S. Mozer, and J. P. Eastwood (2007) Evidence for an elongated ( $>60$ ion skin depths) electron diffusion region during fast magnetic reconnection, Phys. Rev. Lett., 99, 255002, doi:10.1103/PhysRevLett.99.255002.

Pritchett, P. L. (2008), Collisionless magnetic reconnection in an asymmetric current sheet, J. Geophys. Res., 113, A06210, doi:10.1029/ 2007JA012930.

Pritchett, P. L., and F. S. Mozer (2009), Asymmetric magnetic reconnection in the presence of a guide field, J. Geophys. Res., 114, A11210, doi:10.1029/ 2009JA014343.

Retinò, A., et al. (2006), Structure of the separatrix region close to a magnetic reconnection X-line: Cluster observations, Geophys. Res. Lett., 33, L06101, doi:10.1029/2005GL024650.

Roux, A., O. Le Contel, C. Coillot, A. Bouabdellah, B. de La Porte, D. Alison, S. Ruocco, and M. C. Vassal (2008), The search coil magnetometer for THEMIS, Space Sci. Rev., 141, 265-275, doi:10.1007/ s11214-008-9455-8.

Roytershteyn, V., W. Daughton, H. Karimabadi, and F. S. Mozer (2012), Influence of the lower-hybrid drift instability on magnetic reconnection in asymmetric configurations, Phys. Rev. Lett., 108(18), 185001 doi:10.1103/PhysRevLett.108.185001.

Scudder, J. D., R. D. Holdaway, W. S. Daughton, H. Karimabadi, V. Roytershteyn, C. T. Russell, and J. Y. Lopez (2012), First resolved observations of the demagnetized electron-diffusion region of an astrophysical magnetic-reconnection site, Phys. Rev. Lett., 108(22), 225005, doi:10.1103/PhysRevLett.108.225005.

Sonnerup, B. U. O., G. Paschmann, I. Papamastorakis, N. Sckopke, G. Haerendel, S. J. Bame, J. R. Asbridge, J. T. Gosling, and C. T. Russell (1981), Evidence for magnetic field reconnection at the earth's magnetopause, J. Geophys. Res., 86, 10,049-10,067, doi:10.1029/ JA086iA12p10049.

Stenberg, G., T. Oscarsson, M. André, A. Vaivads, M. Morooka N. Cornilleau-Wehrlin, A. Fazakerley, B. Lavraud, and P. M. E. Décréau (2005), Electron-scale sheets of whistlers close to the magnetopause, Ann. Geophys., 23, 3715-3725, doi:10.5194/angeo-23-3715-2005.

Swisdak, M., B. N. Rogers, J. F. Drake, and M. A. Shay (2003), Diamagnetic suppression of component magnetic reconnection at the magnetopause, J. Geophys. Res., 108(A5), 1218, doi:10.1029/ 2002JA009726.

Treumann, R. A., J. Labelle, and R. Pottelette (1991), Plasma diffusion at the magnetopause-The case of lower hybrid drift waves, J. Geophys. Res. 96, 16,009-16,013, doi:10.1029/91JA01671.

Vaivads, A., O. Santolík, G. Stenberg, M. André, C. J. Owen, P. Canu, and M. Dunlop (2007), Source of whistler emissions at the dayside magnetopause, Geophys. Res. Lett., 34, L09106, doi:10.1029/ 2006 GL029195.

Zhang, Y., H. Matsumoto, and H. Kojima (1999), Whistler mode waves in the magnetotail, J. Geophys. Res., 104, 28,633-28,644, doi:10.1029/ 1999JA900301. 